\title{
Mueller matrix polarimetry for improved liver fibrosis diagnosis
}

\author{
Matthieu Dubreuil ${ }^{1, *}$, Philippe Babilotte ${ }^{1}$, Loïc Martin ${ }^{1}$, David Sevrain ${ }^{1}$, Sylvain Rivet ${ }^{1}$, Yann Le \\ Grand $^{1}$, Guy Le Brun ${ }^{1}$, Bruno Turlin ${ }^{2}$ and Bernard Le Jeune ${ }^{1}$ \\ ${ }^{1}$ Université de Brest, UEB, EA 938 Laboratoire de Spectrométrie et Optique Laser, 6 avenue Le Gorgeu, C.S. 93837,29238 \\ Brest Cedex 3, France \\ ${ }^{2}$ Department of Pathology, Hôpital Pontchaillou, 35033 Rennes, France \\ *Corresponding author: matthieu.dubreuil@univ-brest.fr \\ Received Month X, XXXX; revised Month X, XXXX; accepted Month X, \\ XXXX; posted Month X, XXXX (Doc. ID XXXXX); published Month X, XXXX

\begin{abstract}
An experimental Mueller matrix polarimeter is used to quantify human liver fibrosis by measuring retardance and depolarization of thin biopsies. The former parameter is sensitive to fibrillar collagen, the latter is specifically sensitive to fibrillar collagen around blood vessels, which is not significant for liver fibrosis diagnosis. By using depolarization like a filter, retardance distribution enables to distinguish between disease stages and limit the high degree of observer discrepancy. (C) 2012 Optical Society of America

OCIS Codes: $120.2130,120.3930,120.5410,170.3890,170.6935,170.4580$.
\end{abstract}

Human liver fibrosis is characterized by a modification of hepatic tissue with the deposition of fibrillar collagen (type I and III) in excess [1]. The assessment of this disease is mainly established by practiced pathologists from liver biopsies whose extra-cellular matrix (including fibrillar collagen) is stained with a specific dye, usually Sirius red. A five-stage grading system (F0-F4) was thus developed based on a Fibrosis-Metavir scoring system [2]. Grade F0 corresponds to healthy livers, grade F4 to the most aggressive stage (cirrhosis). However, it is currently difficult to obtain highly reproducible results from scores due to the high degree of intra- and inter-observer discrepancy [3]. An effort into quantifying automatically the liver disease has been made but fibrillar collagen that is also abundant around blood vessels in healthy tissues, can distort diagnosis. Thus, image processing methods are necessary to mask fibrotic areas but these processes are complex, generally semi-automatic and time consuming [4]. This Letter demonstrates the potentialities of Mueller polarimetry to first discriminate collagen around vessels from the one associated to fibrosis thanks to depolarizing properties, and then quantify the relevant fibrillar collagen by measuring retardance properties. This study relies on stained samples with Sirius red as it is known that stained fibrillar collagen enhances its natural birefringence [5,6]. A subsequent statistical method is developed to quantify fibrillar collagen with a smaller incidence of human subjectivity and variations in staining.

Mueller matrix (MM) polarimetry is used to obtain the full polarimetric response of samples (depolarization, birefringence and dichroism). The studies have been carried out with a Snapshot Mueller Matrix Polarimeter (SMMP) developed in our lab [7]. Its principle is to encode polarization states in the spectral domain by means of a broadband source and high-order retarders. Thanks to a specific retarder-thickness configuration, the full Mueller matrix of a sample is available in a single spectrum, $I(\lambda)$, measured with a dispersive detection system (spectrometer 1200 grooves $/ \mathrm{mm}$ ).

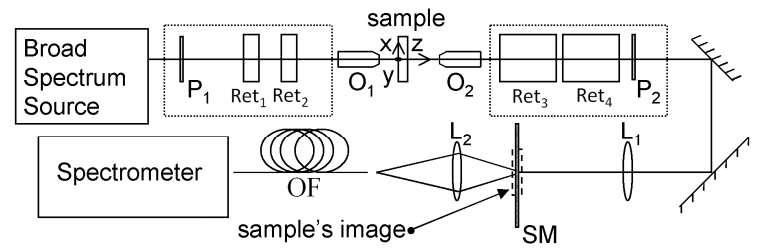

Fig. 1. Diagram of the Snapshot Mueller Matrix Polarimeter setup. $\mathrm{O}_{1}, \mathrm{O}_{2}$ : $10 \mathrm{x}$ objectives, $\mathrm{SM}$ : scattering medium, $\mathrm{L}_{1}, \mathrm{~L}_{2}$ : lenses, OF: optical fiber, other abbreviations defined in text.

The SMMP in Fig. 1 is composed of a 15-nm-broadband source (SLD from B\&W Tek, Inc) emitting around $\lambda_{o}=830 \mathrm{~nm}$, a linear polarizer $\left(\mathrm{P}_{1}\right)$ oriented at $0^{\circ}$, two calcite retarders (Ret $1, \operatorname{Ret}_{2}$ ) of thickness $e=2.08 \mathrm{~mm}$ respectively oriented at $45^{\circ}$ and $0^{\circ}$, two calcite retarders (Ret 3 , Ret 4 ) of thickness $5 e=10.4 \mathrm{~mm}$ respectively oriented at $0^{\circ}$ and $45^{\circ}$, a linear polarizer $\left(\mathrm{P}_{2}\right)$ oriented at $90^{\circ}$, and a spectrometer. The sample under study is imaged on a scattering medium (Bfi Optilas light diffuser, $\left.10^{\circ} \mathrm{FWHW}\right)$ by $\mathrm{L}_{1}$ in order to avoid coherence effects. The signal $I(\lambda)$ is periodic and composed of several frequencies. With this retarder-thickness configuration, 13 frequencies are generated on the $10 \mathrm{~nm}$-analysis window. The coefficients of a Mueller matrix $\left(m_{i j}\right)$ are retrieved through application of a Fourier transform (real part and imaginary part) to $I(\lambda)$. By application of the calibration procedures described in [8], the accuracy on the $m_{i j}$ coefficients (normalized by $m_{00}$ ) is below 0.03 for measurements of well-known media (polarizer, waveplate). The interest of this polarimeter lies essentially in its short acquisition time since it depends only on the detector (aperture time and repetition rate, respectively $10 \mu$ s and $1 \mathrm{kHz}$ in this work).

The parameters issued from experimental Mueller matrices $(M)$ were extracted by the Lu and Chipman decomposition [9], which is based on the assumption that $M$ is the product of three matrices (diattenuation, $M_{D}$, retardance, $M_{R}$, depolarization, $M_{\Delta}$, matrices), 
corresponding to each polarimetric effect (dichroism, birefringence, depolarization) so that $M=M_{\Delta} \cdot M_{R} \cdot M_{D}$. In this study, only the depolarization index, $P_{D}$, calculated directly from $M$ [10], and the retardance, $R$, from $M_{R}$, are analyzed. One should note that the $P_{D}$ index is equal to 1 for a non depolarizing medium and theoretically less than 1 otherwise (although the noise may rise it to above 1). The diattenuation signal, attributed to the Sirius red dye, is weak at the wavelength $830 \mathrm{~nm}$ due to the low absorption of the dye [6]. Moreover, the measurement of the fast axis orientation of the retarder, which gives the direction of collagen fibers, is not considered with here.

We carried out experiments with five $16-\mu \mathrm{m}$ thick acute surgical human liver biopsies that were fixed in formalin and embedded in paraffin. They are then deparaffanized and stained by Sirius red. Samples were mounted between a microscope slide and a $0.17 \mathrm{~mm}$-thick coverslide. The liver histological status was assessed by two trained pathologists, which accounts for the different scores for some biopsies studied in this Letter. Liver cryosections were set in an afocal system built with two $10 \times 0.25 N A$ objectives. Slices were mounted on motorized translation stages in order to scan the samples ( $\mathrm{x}, \mathrm{y}$ directions) and change the cross-section of the beam, i.e. the resolution of the image (by moving samples in $\mathrm{z}$ direction). All of the samples were set at normal incidence by auto-collimation and thus illuminated in the same direction by the laser source. For a $50-\mu \mathrm{m}$ estimated resolution (knife-edge technique), retardance $R$ and depolarization index $P_{D}$ of two biopsies (for example F0 and F4) have been measured and represented pixel by pixel in Fig. 2 for $2.5 \times 2.5 \mathrm{~mm}^{2}$ regions of interest (ROI's). These ROI's were selected from full-field Non-Polarized (NP) images taken with a CMOS camera (not represented on the setup of Fig.1). Second Harmonic Generation (SHG) diffraction-limited images $(2.5-\mu \mathrm{m}$ estimated resolution) of the same ROI's were obtained on the scanning nonlinear microscope of our lab (Olympus BX51WI-FV300 confocal system equipped with a Coherent Mira-Verdi femtosecond laser tuned at 830nm and a home-built SHG photodetector at $415 \mathrm{~nm}$ settled in transmission) using a low-NA objective (Olympus UPLSAPO $4 \times$ 0.16NA).

In the classical Non-Polarized image of the F4 biopsy, fibrotic areas appear darker (due to Sirius dye) than the normal tissue. Similar structures are observed on the retardance image, which is also closely correlated to the corresponding SHG image. As SHG imaging is highly specific to fibrillar collagen [11], we can conclude that retardance is mainly due to fibrillar collagen. On the other hand, depolarization index slightly changes on the ROI and is close to 1 in the F4 biopsy. However if $R$ and $P_{D}$ are analyzed in the area of blood vessels (for example in the F0 biopsy which possess a lot of vessels), results are quite different. As expected, retardance appears in the vessel region due to fibrillar collagen (confirmed by the SHG image), but there is also a stronger depolarization effect (i.e. depolarization index less than 1) when the laser beam focuses on the vessel walls. In this case, there is an important spatial variation of retardance within the laser beam cross section (one part of the beam sees birefringence and the other part sees no polarization effects). The polarimetric properties distributed in the beam are incoherently added, leading to depolarization. This effect has been observed on every vessel regions, whatever the degree of the disease. This could be explained by the fact that on vessels walls, the spatial variation of retardance is the strongest. Thus fibrotic collagen could be distinguished from collagen around vessels thanks to depolarization index. One should note that given the thickness of the biopsies $(16 \mu \mathrm{m})$, depolarization due to forward scattering is negligible (scattering mean free path $\approx 100 \mu \mathrm{m}$ ).

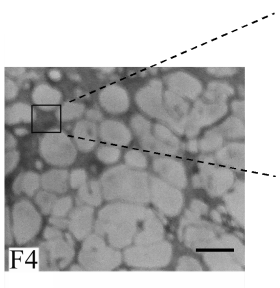

NP image

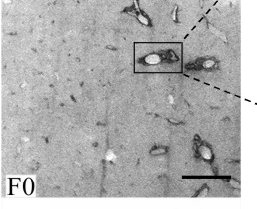

NP image

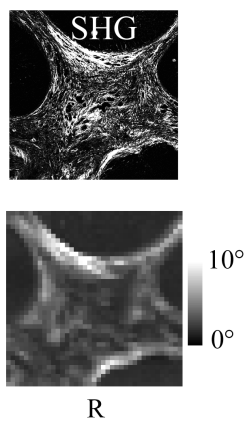

$\mathrm{R}$

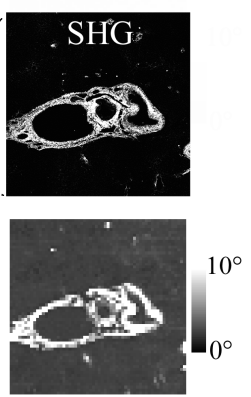

$\mathrm{R}$

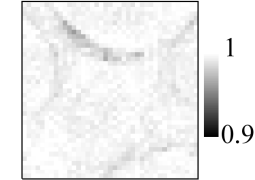

$\mathrm{Pd}$

$\operatorname{Pd}$

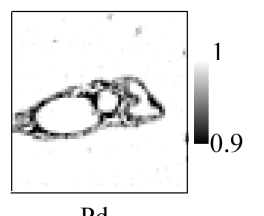

Fig. 2. Retardance $(R)$ and depolarization index $\left(P_{D}\right)$ images of $2.5 \times 2.5 \mathrm{~mm}^{2}$ ROI's of stained surgical human liver biopsies (F0 and F4 Metavir grades). Images were obtained with a 50- $\mu \mathrm{m}$ estimated resolution and a 40- $\mu \mathrm{m}$ step size. For comparison, SHG images of the same ROI's were done with a 2.5- $\mu$ m estimated resolution, under circular illumination. The Non-Polarized (NP) images of the global biopsies are also shown, and the scale bars are equal to $0.25 \mathrm{~cm}$.

Then, after using images to assign depolarization to the collagen of vessels, a statistical method has been developed in order to quantify the grade of the disease without building any image. Retardance and depolarization index were measured by scanning, with a $50-\mu \mathrm{m}$ estimated resolution, a surface of $1.5 \times 1.5 \mathrm{~cm}^{2}$ area with a $250-\mu \mathrm{m}$ step size (i.e. a sampling of about 3600 measurements on the whole liver biopsies). The depolarization index $P_{D}$ is used to filter retardance values due to collagen around blood vessels and select only those resulting from the modification of hepatic tissue by keeping measurements with experimental $P_{D}$ values ranging from 0.98 to 1.02 , due to Gaussian noise. Every time the laser beam probes a diseased area, the retardance value is superior to the baseline (Fig. 3).

The probability distribution of retardance is assessed for the samples after $P_{D}$ filtering and is different from a Gaussian distribution according to the modification of the hepatic tissues. The retardance distribution is 
characterized by the mean, $R_{0}$, and a retardance value $R_{\text {lim }}$ for which the frequency of the theoretical Gaussian distribution is equal to $1 \%$ of the maximum. It can be noted that $R_{0}$ and $R_{\text {lim }}$ depend on: 1) the stability of the room temperature which slightly modifies retardance measurements, 2) the beam focusing conditions and 3) the staining variability.
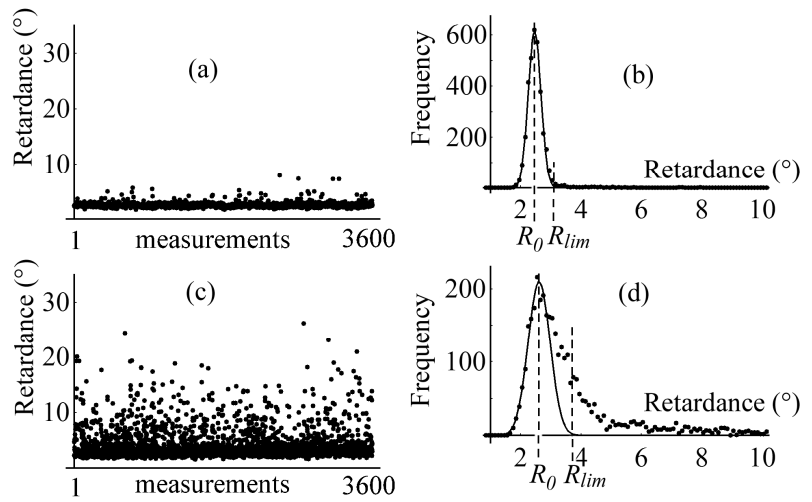

Fig. 3. Retardance values obtained by a F0 grade liver (a) and a F4 grade liver (c) on a surface of $1.5 \times 1.5 \mathrm{~cm}^{2}$ area with a $250-\mu \mathrm{m}$ step size (3600 measurements), after $P_{D}$ filtering. (b) and (d) Experimental distribution of retardance values respectively for F0 and F4 grade liver (filled circles). Data were fitted by a Gaussian curve (solid curve) for the weaker retardance values.

In order to evaluate human liver fibrosis, two indexes $\bar{R}$ and $N$ are defined to quantify the distribution of retardance beyond $R_{\text {lim }}$, by the following relationships:

$$
\bar{R}=\frac{1}{N_{\text {sup }}} \sum_{R>R_{\text {lim }}} R \quad \text { (1) } \quad N=\frac{N_{\text {sup }}}{N_{\text {tot }}}
$$

where $N_{\text {sup }}$ is the number of measurements for which retardance is greater than $R_{\text {lim }}$, and $N_{\text {tot }}$ the number of measurements for each sample. $R_{\text {lim }}$ and $R_{0}$ being values calculated for each sample, $\bar{R} / R_{0}$ and $N$ should be less sensitive to the versatility of experimental protocols (staining, thickness). Samples are plotted on a $2 \mathrm{D}$ graph according to $N$ and $\bar{R} / R_{0}$ (Fig. 4 ).

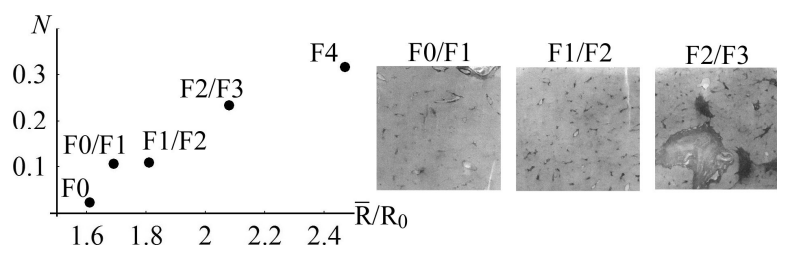

Fig. 4. Plot of liver samples according to $N$ index and $\bar{R} / R_{0}$. The Non-Polarized (NP) images of 3 biopsies are also presented. Samples are named according to the disease grade measured by two pathologists, which accounts for the double notation "F0/F1", "F1/F2" and "F2/F3".

$\bar{R} / R_{0}$ seems to be a good differentiator since its value depends on the amount of fibrillar collagen, and it avoids any ambiguity of diagnosis after applying the $P_{D}$ filter. Moreover the index $N$ is also interesting because it is sensitive to the spatial expanse of the deposit of collagen. For instance the samples F0/F1 and F1/F2 show the same pattern, and their respective $N$ indices are similar. Similarly when the area of the disease increases, the $N$ index rises (samples F2/F3 and F4).
The choice of the beam focus can be a relevant element in order to enhance retardance values and obtain a more sensitive measurement. Indeed due to the spatial inhomogeneity of the birefringence properties (discrepancy of optical axis directions), only the mean retardance is measured and it increases with the focusing as Fig. 5 shows. Nevertheless, if the spot size drops to below $50 \mu \mathrm{m}$ when the sample is moved between the two objectives, the image background increases. The origin of this modification is not clear, and could come from the residual paraffin in which liver specimens were first embedded [12].

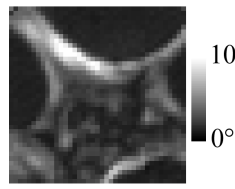

(a)

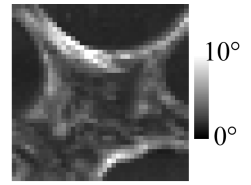

(b)

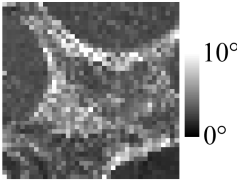

(c)
Fig. 5. Retardance image of F4 grade liver. (a) Spot size $>50 \mu \mathrm{m}$, (b) spot size $\approx 50 \mu \mathrm{m}$, (c) spot size $<50 \mu \mathrm{m}$.

In conclusion, the present paper illustrates and discusses a novel automated quantitative method based on a scanning system that measures the polarimetric properties of hepatic tissues. This tool is able to quantify the fibrillar collagen of liver fibrosis without scoring the fibrillar collagen around vessels thanks to depolarization. The study of retardance distribution after filtering enables to distinguish between disease stages and should limit the high degree of observer discrepancy. Thus, our method represents a complement to semi-quantitative indexes of fibrosis to define the evolution stage of several hepatic diseases more accurately.

This work is financially supported in part by the "Conseil Général du Finistère".

\section{References}

1. R. Bataller and D.A. Brenner, J. Clin. Invest. 115, 209-218 (2005).

2. P. Bedossa and T. Poynard, Hepatology 24(2), 289-293 (1996).

3. The French METAVIR Cooperative Study Group, Hepatology 20(1), 15-20 (1994).

4. G.M. Dahab, M. M. Kheriza, H.M. El-Beltagi, A.M. Fouda and O.A. El-Din, J. Gastroenterol. Hepatol 19(1), 78-85 (2004)

5. L.C.U. Junqueira, G. Bignolas and R.R. Brentani, Histochem. J. 11, 447-155 (1979).

6. B. Laude-Boulesteix, A. De Martino, B. Drévillon and L. Schwartz, Appl. Opt. 43, 2824-2832 (2004).

7. M. Dubreuil, S. Rivet, B. Le Jeune and J. Cariou, Opt. Expr. 15, 13660-13668 (2007).

8. M. Dubreuil, S. Rivet, B. Le Jeune and J. Cariou, Appl. Opt. 48, 1135-1142 (2009).

9. S.-Y. Lu and R.A. Chipman, J. Opt. Soc. Am. A 13, 11061113 (1996).

10. J.J. Gil and E. Bernabeu, J. Mod. Optic. 33, 185-189 (1986).

11. L. Gaihouste, Y. Le Grand, C. Odin, D. Guyader, B. Turlin, F. Ezan, Y. Désille, T. Guilbert, A. Bessard, C. Frémin, N. Theret and G. Baffet, J. Hepatol. 52, 398 406 (2010).

12. K. Kiraly, M.M. Hyttinen, T. Lapvetelainen, M. Elo, I. Kiviranta, J. Dobai, L. Modis, H.J. Helminen and J.P.A. Arokoski, J. Mol. Histol. 29, 317-327 (1997). 


\section{Full references}

1. R. Bataller and D.A. Brenner, "Liver fibrosis," Journal of Clinical Investigation 115, 209-218 (2005).

2. P. Bedossa and T. Poynard, "An algorithm for the grading of activity in chronic hepatitis C. The METAVIR Cooperative Study Group," Hepatology 24(2), 289-293 (1996).

3. The French METAVIR Cooperative Study Group, "Intraobserver, interobserver variations in liver biopsy interpretation in patients with chronic hepatitis C", Hepatology 20(1), 15-20 (1994)

4. G.M. Dahab, M.M. Kheriza, H.M. El-Beltagi, A.M. Fouda and O.A. El-Din, "Digital quantification of fibrosis in liver biopsy sections: description of a new method by Photoshop software," Journal of Gastroenterology and Hepatology 19(1), 78-85 (2004)

5. L.C.U. Junqueira, G. Bignolas and R.R. Brentani, "Picrosirius staining plus polarization microscopy, a specific method for collagen detection in tissue sections," Histochemical Journal 11, 447-155 (1979).

6. B. Laude-Boulesteix, A. De Martino, B. Drévillon and L. Schwartz, "Mueller polarimetric imaging system with liquid crystals," Applied Optics 43, 2824-2832 (2004).

7. M. Dubreuil, S. Rivet, B. Le Jeune and J. Cariou, "Snapshot Mueller matrix polarimeter by wavelength polarization coding," Optics Express 15, 13660-13668 (2007).

8. M. Dubreuil, S. Rivet, B. Le Jeune and J. Cariou, "Systematic errors specific to a snapshot Mueller matrix polarimeter," Applied Optics 48, 1135-1142 (2009).

9. S.-Y. Lu and R.A. Chipman, "Interpretation of Mueller matrices based on polar decomposition," Journal of the Optical Society of America A 13, 1106-1113 (1996).

10. J.J. Gil and E. Bernabeu, "Depolarization and polarization indices of an optical system," Journal of Modern Optics 33, 185-189 (1986).

11. L. Gaihouste, Y. Le Grand, C. Odin, D. Guyader, B. Turlin, F. Ezan, Y. Désille, T. Guilbert, A. Bessard, C. Frémin, N. Theret and G. Baffet, "Fibrillar collagen scoring by second harmonic microscopy: A new tool in the assessment of liver fibrosis," Journal of Hepatology 52, 398-406 (2010).

12. K. Kiraly, M.M. Hyttinen, T. Lapvetelainen, M. Elo, I. Kiviranta, J. Dobai, L. Modis, H.J. Helminen and J.P.A. Arokoski, "Specimen preparation and quantification of collagen birefringence in unstained sections of articular cartilage using image analysis and polarizing light microscopy," Journal of Molecular Histology 29, 317-327 (1997). 